\title{
Associations between C-section and hand grip strength: an observational analysis and genome-wide environmental interaction study
}

\author{
Xiaomeng $\mathrm{Chu}^{1}$, Li Liu ${ }^{1}$, Jing $\mathrm{Ye}^{1}$, Yan Wen ${ }^{1}$, Ping $\mathrm{Li}^{1}$, Bolun Cheng ${ }^{1}$, Shiqiang Cheng ${ }^{1}$, \\ Lu Zhang ${ }^{1}$, Xin Qi ${ }^{1}$, Mei Ma ${ }^{1}$, Chujun Liang ${ }^{1}$, Om Kafle ${ }^{1}$, Cuiyan Wu ${ }^{1}$, Sen Wang ${ }^{1}$, Xi \\ Wang $^{1}$, Yujie Ning ${ }^{1}$, and Feng Zhang ${ }^{1}$ \\ ${ }^{1}$ Affiliation not available
}

October 22, 2020

\begin{abstract}
Objective: Hand grip strength (HGS) could be taken as a biomarker for overall health of individual. While growing evidence suggests cesarean section (C-section) related to child health, few studies have assessed its effect on the overall health status of offspring. Design: Cross-sectional cohort study. Setting: UK Biobank cohort. Population: A population-based cohort consisting of 160,635 participants for left HGS and 160,651 for right HGS aged between 40 and 69. Methods: Regression analyses were conducted to test the associations between C-section and left HGS and right HGS. Then, genome-wide of environment interaction study (GWEIS) was conducted by PLINK 2.0 to identify loci with gene-environment $(\mathrm{G} \times \mathrm{E})$ interaction effects, using C-section as an environmental factor. FUMA platform was used for functional gene set enrichment analysis of identified candidate genes. Main Outcome Measures: C-sections and hand grip strength. Results: The regression analyses showed significant associations $(\mathrm{P}<0.001)$ between $\mathrm{C}$-section and left and right HGS. GWEIS detected 6 significant G $\times$ E interaction effects on left HGS, such as ADGRV1 gene, POLR3G gene, and WASL gene. 32 significant $\mathrm{G} \times \mathrm{E}$ interactions effects on right HGS were detected, such as APBB2 gene, and CRPPA gene. Comparing the analyses results of left HGS and right HGS, identified suggestive significant interactions between CLEC16A gene and C-section, such as rs80001954 (Pright = 5.21 x 10-8, Pleft = 1.22 x 10-7); rs117658390 (Pright $=5.43 \times 10-8$, Pleft $=1.09 \times 10-7)$ and rs79479146 (Pright $=5.45 \times 10-8$, Pleft $=1.22 \times 10-7)$. Gene set enrichment analyses reported 12 GO terms, such as GO_CENTRAL_NERVOUS_SYSTEM_DEVELOPMENT (adjusted P $=1.98 \times 10-3$ ), and GO_HEAD_DEVELOPMENT (adjusted $\mathrm{P}=5.09 \times 10-3$ ). Conclusion: Our study holds potential for clarifying the functional relevance of C-section with HGS
\end{abstract}

\section{Introduction}

Hand grip strength (HGS), as one of the simplest and least complicated of instrumented muscle strength measures, is showed to reflect overall muscle strength ${ }^{1}$. Decreased muscle strength and functionality are common and complex changes related to ageing and could affect the function of daily life and survival activities, which increases personal and societal costs ${ }^{2}$. The measurement of HGS is simple and reliable and it is related to the function of daily life and survival activities. Therefore, HGS has been used as a significant predictor and biomarker of overall health of individual, not only in the elderly, but also in middleaged and young people. A number of studies have demonstrated the predictive role of HGS in complicated diseases and traits. For example, Firth et al. reported significant positive associations between maximal HGS and improved cognitive performance in people with major depression and bipolar disorder ${ }^{3}$. Leong et al. ${ }^{4}$ performed a prospective epidemiology study including 7 countries and showed that HGS predicted not only all-cause mortality but also cardiovascular mortality, non-cardiovascular mortality, cardiovascular disease occurrence and stroke. In addition, the associations between HGS and mortality were observed not only in 
older but also in middle-aged and young adults ${ }^{4}$. Given the reliable and accuracy of HGS as an indicator of general health and nutritional status, we use HGS as a biomarker for overall health of individual in this study.

The heritability of grip strength estimated by Finkel et al. was approximately $75 \%$ in men and $47 \%$ in women ${ }^{5}$. Sara et al. performed a large-scale genome-wide association study (GWAS) analysis and reported 16 loci associated with grip strength containing genes implicated in structure and function of skeletal muscle fibers, neuronal maintenance and signal transduction, or psychomotor impairment ${ }^{6}$. What's more, Inge et al. ${ }^{7}$ observed a significant gene-environment $(\mathrm{G} \times \mathrm{E})$ interaction effect on grip trajectories, suggesting that in addition to genetic factors, $\mathrm{G} \times \mathrm{E}$ interactions also influence HGS. While the effects of genetics and $\mathrm{G} \times \mathrm{E}$ interactions on HGS have been proved, the extent and mechanisms of their effects are still unclear.

Cesarean section (C-section) is a lifesaving procedure when delivery is obstructed, or the fetus is in poor condition, and is effective in reducing neonatal and maternal mortality ${ }^{8}$. The rate of C-section has increased more than 3 -fold worldwide during the past 30 years ${ }^{8}$. It is of concern that C-section have been shown to have potential long-term health implications on offspring. C-section could lead to negative health outcomes including allergies ${ }^{9}$, asthma ${ }^{9}$, and metabolic abnormalities ${ }^{10}$. C-section, act as an environment risk factor, might disturb immune activation in offspring by reducing hypothalamus-pituitary-adrenal axis activation ${ }^{11}$, and alter the bacterial colonization through the lack of exposure to maternal bowel flora ${ }^{12}$. While growing evidence suggests that C-section related to child health, less study have assessed its effect on the overall health status of offspring.

Genome-wide by environmental interaction study (GWEIS) is a method taking into account the role of $\mathrm{G} \times \mathrm{E}$ interaction. $\mathrm{G} \times \mathrm{E}$ interaction indicates the effect due to a mixture of environmental factors and genetic factors, playing an important role in identifying the mechanism of a lot of complex traits and diseases ${ }^{13}$. In contrast to GWAS, GWEIS included genome-wide data rather than candidate genes, which significantly improved the ability to detect genotype-phenotype associations missed in GWAS ${ }^{14}$.

In this study, we aimed to assess whether C-section constitutes a Gene× C-section interaction with genetic factors and thereby affecting HGS in offspring. Observational analyses followed by GWEIS were performed using individual phenotype and genotype data from UK Biobank. Then we performed gene-set enrichment analysis using FUMA platform. Our study holds potential for clarifying the functional relevance of C-section with HGS.

\section{Method}

\section{Phenotype definition}

Phenotypic data in this study were derived from UK Biobank, a population-based cohort consisting of 502,641 participants aged between 40 and $69^{15}$. Participants underwent a baseline assessment capturing sociodemographic and health status, including age, sex, BMI and HGS (both left and right). Jamar dynamometer was used for the assessment of left HGS and right HGS ${ }^{16,17}$. C-sections were self-reported at the nurse-validated baseline assessment.

Genotyping, imputation and quality control

The UK Biobank cohort consists of about 500,000 participants, with 487,409 individuals having genetic data. Genotyping, quality control and imputation were performed by the UK Biobank ${ }^{1518}$. In brief, the Affymetrix UK BiLEVE Axiom and the Affymetrix UK Biobank Axiom arrays (Santa Clara, CA, USA) were used for genotyping. The two have over $95 \%$ of content in common. Imputation was carried out by IMPUTE4 in chunks of approximately 50,000 imputed markers with a $250 \mathrm{~kb}$ buffer region. Imputation reference panel included the 1000 Genomes phase 3 dataset, the merged UK10K and 1000 Genomes phase 3 reference panels and the Haplotype Reference Consortium (HRC) data. Quality control (QC) consisted of two parts, named sample-based QC and marker-based QC, and they were performed using PLINK v1.9 and $\mathrm{R}$ v3.3.1. KING software was used to generate unrelated subjects. It includes a rapid algorithm for relationship inference that allows the presence of unknown population substructure ${ }^{19}$. Missing rate 
and heterozygosity were used to identify the poor quality samples in the sample-based QC. Statistical tests of batch effects, plate effects, Hardy-Weinberg equilibrium, sex effects, array effects, and discordance across control replicates were performed to identify poor quality markers in marker-based QC, checking for consistency across experimental factors. Detailed information of the genotyping, imputation and quality control could be found in the published study ${ }^{18}$.

Observational analyses

The observational analyses were performed using $\mathrm{R}$ software. We firstly used T-test to estimate the differences in age, BMI, and HGS between male and female in the study population. Then regression analyses was performed to evaluate the associations between C-section and left HGS ( $\mathrm{n}=160,635)$ and right HGS $(\mathrm{n}=160,651)$, respectively. Age, sex, BMI and 10 population structure principal components (PCs, calculated by UK Biobank) were used as covariates.

GWEIS analyses

GWEIS were conducted by PLINK2.0 ${ }^{20}$ for HGS using C-section as an environmental factor. This method considers SNP-environment interaction effects from a regression model, which has been shown that it has more power to detect genetic effects than does a test of association between disease and the genetic variant ${ }^{21}$. The quality control was assessed for each sample at the SNP levels. SNPs with low call rates $(<0.90)$, low Hardy-Weinberg equilibrium exact test $P$ values $(<0.001)$, or low minor allele frequencies $($ MAFs $<0.01)$ were excluded. For every single locus, there are two models in the previous gnome-wide association studies, called additive model and dominance model. In this study, to consider the role of C-section, we tested $\mathrm{SNP}_{\mathrm{ADD}} \mathrm{xC}$-section model for each SNP. Significant SNPs were identified at $P<5.00 \times 10^{-8}$.

Gene set enrichment analysis

FUMA platform (http://fuma.ctglab.nl/) was used to study the enrichment analysis of the obtained genes. FUMA is a web-based comprehensive platform that can utilize a variety of biological resource information to annotate the function, gene priority and interactive visualization of GWAS results ${ }^{22}$. Genes with genomewide significant interaction effects or suggestively significant interaction effects on left HGS or on right HGS identified in GWEIS were used as input. GENE2FUNC function was used to obtain related gene ontology (GO). Enrichment of prioritized genes is tested using the hypergeometric test against gene sets obtained from MsigDB ${ }^{23}$. A total of $5817 \mathrm{GO}$ terms were included in the analyses, in which 4436 for GO biological process, 580 for GO cellular component and 901 for GO molecular function. Benjamini-hochberg method was performed to correct multiple tests.

Data visualization

Circular Manhattan plots were generated using the "CMplot" R script (https://github.com/YinLiLin/RCMplot).

Ethical approval

The signed consents were provided in the participants visit assessment. Ethical approval of UK Biobank was granted by the National Health Service National Research Ethics Service (reference 11/NW/0382).

\section{Result}

Association between C-section and HGS in the UKB

Population features are shown in Table 1. 160635 and 160651 participants were included in the observational analyses of left HGS and right HGS, respectively. There were significant differences in age, BMI and grip strength $(P<0.001)$, as the male participants had higher mean values than the females. Regression analyses showed significant associations between C-section and left HGS $\left(\beta=-0.77, P<0.001\right.$, adjusted $\left.R^{2}=0.5699\right)$ and right HGS $\left(\beta=-0.72, P<0.001\right.$, adjusted $\left.R^{2}=0.5645\right)$ (Table 2$)$.

GWEIS analyses 
All the significant $\mathrm{G} \times \mathrm{E}$ interactions $\left(P<5.00 \times 10^{-8}\right)$ and suggestive significant $\mathrm{GxE}$ interactions $(P<1.00$ $\mathrm{x} 10^{-5}$ ) were shown in Supplemental Table 1-2. For left HGS, we detected 6 significant GxE interactions of Csection, including ADGRV1 gene $\left(\mathrm{rs} 77788156, P=9.13 \times 10^{-9} ; \mathrm{rs} 114592446, P=2.16 \times 10^{-8}\right)$, POLR3G gene $\left(\right.$ rs112985393, $\left.P=9.29 \times 10^{-9}\right)$, and WASL gene $\left(r s 62472074, P=4.74 \times 10^{-8}\right)$ (Supplemental Table 1). For right HGS, 32 significant GxE interactions of C-section were detected, including APBB2 gene (rs62409974, $P$ $\left.=2.70 \times 10^{-8} ; \mathrm{rs} 62410025, P=2.89 \times 10^{-8} ; \mathrm{rs} 62409998, P=2.93 \times 10^{-8}\right)$, and CRPPA gene $(\mathrm{rs} 148803065, P$ $\left.=1.32 \times 10^{-8}\right)$. Several SNPs nearing CRPPA gene were also showed suggestive significant interactions with C-section (Supplemental Table 2). Interestingly, Comparing the analyses results of left HGS and right HGS, identified suggestive significant interactions between CLEC16A gene and C-section, including rs80001954 $\left(P_{\text {right }}=5.21 \times 10^{-8}, P_{\text {left }}=1.22 \times 10^{-7}\right) ; \operatorname{rs} 117658390\left(P_{\text {right }}=5.43 \times 10^{-8}, P_{\text {left }}=1.09 \times 10^{-7}\right)$ and rs79479146 $\left(P_{\text {right }}=5.45 \times 10^{-8}, P_{\text {left }}=1.22 \times 10^{-7}\right)($ Supplemental Table 3$)$.

Functional gene set enrichment analysis

Gene set enrichment analyses reported 12 GO terms (Table 3, Figure 1), such as GO_CENTRAL_NERVOUS_SYSTEM_DEVELOPMENT (adjusted $P=1.98 \times 10^{-3}$ ), GO_HEAD_DEVELOPMENT (adjusted $P=5.09$ x $10^{-3}$ ) and GO_CIRCULATORY_SYSTEM_DEVELOPMENT (adjustedP $=2.48 \times 10^{-2}$ ).

\section{Discussion}

To the best of our knowledge, the present study is the first population-scale study to investigate relationships between HGS and C-section. We performed observational analysis and GWEIS using over 160,000 participants from the UK Biobank. We showed significant associations between HGS and C-section and proved several GenexC-section interactions effecting on HGS.

Our observational analysis proved a significant association between C-section and HGS in offspring. Based on previous studies, we hypothesized that this association might be related to the changes caused by Csection in intestinal flora ${ }^{24}$ and immune responses in offspring ${ }^{25}$. These changes might act as prenatal environmental factors that have long-term or permanent effects on skeletal muscle growth and decline in offspring ${ }^{26}$. Previous studies have observed the cross talk between gut microbiota and skeletal muscle in pathological conditions ${ }^{27}$ and proposed that intestinal flora regulates skeletal muscle function through skeletal muscle-gut axis ${ }^{27,28}$. Lahiri et al. ${ }^{29}$ observed that the mice lacked gut microbiota had skeletal muscle atrophy, decreased expression of insulin-like growth factor 1, and reduced transcription of genes associated with skeletal muscle growth and mitochondrial function. These phenomena could be improved by transplanting intestinal flora from pathogen-free mice into germ-free mice ${ }^{29}$. A population-based study found that C-section lead to a decrease in the abundance of Bifidobacteriumin the intestines of infants ${ }^{30}$. Bifidobacterium may influence gut-muscle communication and regulate muscle size ${ }^{31}$. Data showed that treatment which could increase the abundance of Bifidobacterium in older individuals may prophylactically moderate aging muscle loss ${ }^{31}$. In addition, immune responses also might be implicated in the mechanism of this association between C-section and HGS. Lipopolysaccharide (LPS), sitting at the neonatal immune system priming, was significantly under-represented in C-section neonates ${ }^{32}$, which may result in persistent effects on human physiology in later life ${ }^{33}$. Immune responses and related factors play a role in skeletal muscle formation. For example, the TGF- $\beta$ family members are implicated in the regulation of myogenic differentiation and have been shown to be potent inhibitors of myoblasts differentiation ${ }^{34}$. These results suggested a role for the gut microbiota and immune system in regulating skeletal muscle mass and function.

ADGRV1 gene showed a significant $G \times E$ interaction effect on left grip strength. ADGRV1 encodes a member of the G-protein coupled receptor superfamily and act as an important regulator of bone homeostasis and muscle function directly or indirectly. Mutations in this gene contribute to epilepsy with myoclonic seizures 35. Mouse models and qRT-PCR analysis demonstrated that ADGRV1-deficient osteoblasts have increased RANKL expression, which result in low $\mathrm{BMD}^{36}$. RANKL as a member of the TNF receptor superfamily is essential for osteoclastogenesis, and its activation induces high-turnover osteoporosis ${ }^{37}$. In addition, RANKL is also expressed in skeletal muscle and activates the NF- $x \mathrm{~B}$ pathway, which inhibits myogenic differentiation and leads to skeletal muscle dysfunction and $\operatorname{loss}^{38}$. Inhibition of RANKL expression could improve muscle 
strength in osteoporotic mice and humans ${ }^{39}$. Previous studies have reported muscle-bone interactions and possible bone-to-muscle communication ${ }^{40}$. Our result supported previous findings and suggested that the effects of C-section on hand grip might be related to muscle-bone interactions.

POLR3G gene and WASL gene showed significant $\mathrm{G} \times \mathrm{E}$ interaction effects on left grip strength while CRPPA gene and APBB2 gene showed significant $\mathrm{G} \times \mathrm{E}$ interaction effects on right grip strength. POLR3G participants in the transcription of RNA polymerase III (Pol III) and express in the skeletal muscle cell lineage, which results in a significant reduction in the activation of muscle genes ${ }^{41}$. In addition, the POLR3G overexpression had some ability to reverse differentiation ${ }^{41}$. WASL gene encodes a member of the Wiskott-Aldrich syndrome (WAS) protein family named N-WASP, which is expressed ubiquitously ${ }^{42}$. WASL could promote actin polymerization and the formation of branched actin polymers which plays an important role in the differentiation of myoblast to myotubes ${ }^{43}$. Cai et al. suggested that N-WASP is a key intermediate in the regulation of myofibroblast differentiation and maturation ${ }^{44}$. CRPPA gene, locating at chromosome $7 \mathrm{p} 21$, is a frequent cause of Walker-Warburg syndrome ${ }^{45}$. Although the functional role of human CRPPA remains unknown, Riemersma et al. ${ }^{46}$ suggested that CRPPA deficiency contribute to tertiary dystroglycanopathies, which is strongly associated with congenita muscular dystrophy $(\mathrm{CMD})^{45}$. APBB2 gene encodes a kind of cytoplasmic adaptor protein which mediates the assembly of multimolecular complexes ${ }^{47}$. APBB2 knockout (KO) mice showed several phenotypes in muscle that are comparable to the clinical features of CMD in humans, including motor impairments and neuromuscular junction (NMJ) abnormalities ${ }^{47} 48$.

Interestingly, gene set enrichment analyses results suggested a role of immune system involved in the association between C-section and HGS. The relationship between C-section and immunity has been discussed above. In addition, the relationship between immunity and HGS has been observed in a monozygotic-twins epigenome-wide study ${ }^{49}$. CLEC16A gene was reported as a candidate gene associated with both left and right HGS in present study. CLEC16A gene encodes a member of the C-type lectin domain containing family and has been showed to be associated with susceptibility for various autoimmune diseases, such as rheumatoid arthritis and systemic lupus erythematous (SLE) ${ }^{50}$. One of the possible potential functional links between CLEC16A and autoimmunity is autophagy ${ }^{51}$, which has been showed to have crosstalks with signaling cascades in both innate and adaptive immune responses ${ }^{52}$. According to a quantitative proteomics analysis, Tam et al. ${ }^{51}$ identified that CLEC16A may enhance mTORC1 activity and act as a negative regulator through mTOR pathway in autophagy. The mTOR is a serine/threonine kinase capable of integrating several stimuli from the medium and growing evidence suggests that mTOR signaling pathway plays an important role in maintaining muscle fiber size in healthy muscle ${ }^{53}$. For example, mTORC1 could mediate the signaling of substrates and control the initiation of translation by which consequently promote the synthesis of muscle protein ${ }^{54}$.

What's more, the results of gene set enrichment analysis suggested the role of central nervous system (CNS) in the relationship between C-section and HGS. Besides the muscle structure, the ability of muscle to produce force is also associated with neuromotor control. Evidence showed that the primary motor cortex (M1) contributes to the control of a power grip of finger muscles during a variety of grasping behaviors ${ }^{55}$. Kara et al. demonstrated that neuromotor control dysfunction contributes to the deterioration of the force generating capacity of skeletal muscles ${ }^{56}$. More research is needed to verify this hypothesis.

One of the innovations of this study is to consider the $\mathrm{G} \times \mathrm{E}$ interaction effects on hand grip strength, which has been demonstrated to contribute to the etiology of complex traits. The GWEIS analyses complement the genetic relationships between C-section and HGS and provide several novel loci contributing to the variation of HGS. In addition, the large samples of HGS in UK biobank increased the accuracy and effectiveness of our analyses. Previous studies focused on the effect of C-section on child health, less study have assessed its effect on the overall health status of offspring. Our study demonstrated the interaction association between C-section and the HGS for offspring. As far as we known, this is the first systemic study exploring the effect of C-section as environmental factor on HGS for offspring. Our study holds great potential for clarifying the functional relevance of C-section with HGS.

Admittedly, there are still limitations in present study. First, because of the sample population, our results 
cannot be generalized across ethnic groups. Secondly, although several genes have been reported to have interaction effects with $\mathrm{C}$-section, the specific biological functions and mechanisms of action need further experimental studies.

In summary, we performed an observational analysis and a genome-wide interaction analysis of C-section and HGS. We reported significant interactions whether from phenotypic or genetic perspective. Our study reported several novel candidate loci and indicated the role of C-section in HGS.

\section{Disclosure of interest}

Authors reported no biomedical financial interests or potential conflicts of interest.

\section{Contribution to authorship}

Author Contributions: Miss Chu had full access to all the data in the study and takes responsibility for the integrity of the data and the accuracy of the data analysis. Dr Liu contributed equally to the work as co-senior authors.

Concept and design: Chu, Liu and Zhang.

Acquisition, analysis, or interpretation of data: Chu, Liu, Qi, Ma, Zhang.

Drafting of the manuscript: Chu and Liu.

Critical revision of the manuscript for important intellectual content: Wen, $\mathrm{Li}$,

Cheng, Liang, Kafle, Wu, Wang and Chu

Statistical analysis: Ye and Chu.

Administrative, technical, or material support: Ye, Wen and Zhang

Supervision: Zhang

\section{Details of ethics approval}

The signed consents were provided in the participants visit assessment. Ethical approval of UK Biobank was granted by the National Health Service National Research Ethics Service (reference 11/NW/0382).

\section{Funding}

This work was supported by the National Natural Scientific Foundation of China [81472925, 81673112]; the Key projects of international cooperation among governments in scientific and technological innovation [2016YFE0119100]; the Natural Science Basic Research Plan in Shaanxi Province of China [2017JZ024]; and the Fundamental Research Funds for the Central Universities.

\section{Reference}

1. Bohannon RW. Muscle strength: clinical and prognostic value of hand-grip dynamometry. Current Opinion in Clinical Nutrition \& Metabolic Care. 2015;18(5).

2. Marzetti E, Calvani R, Tosato M, Cesari M, Di Bari M, Cherubini A, et al. Sarcopenia: an overview. Aging Clinical and Experimental Research. 2017 2017/02/01;29(1):11-7.

3. Firth J, Firth JA, Stubbs B, Vancampfort D, Schuch FB, Hallgren M, et al. Association Between Muscular Strength and Cognition in People With Major Depression or Bipolar Disorder and Healthy Controls. JAMA Psychiatry. 2018;75(7):740-6.

4. Sayer AA, Kirkwood TBL. Grip strength and mortality: a biomarker of ageing? The Lancet. 2015 2015/07/18/;386(9990):226-7.

5. Finkel D, Pedersen NL, Reynolds CA, Berg S, de Faire U, Svartengren M. Genetic and Environmental Influences on Decline in Biobehavioral Markers of Aging. Behavior Genetics. 2003 2003/03/01;33(2):107-23. 
6. Willems SM, Wright DJ, Day FR, Trajanoska K, Joshi PK, Morris JA, et al. Large-scale GWAS identifies multiple loci for hand grip strength providing biological insights into muscular fitness. Nat Commun. 2017;8:16015-.

7. Petersen I, Pedersen NL, Rantanen T, Kremen WS, Johnson W, Panizzon MS, et al. GxE Interaction Influences Trajectories of Hand Grip Strength. Behavior genetics. 2016;46(1):20-30.

8. Boerma T, Ronsmans C, Melesse DY, Barros AJD, Barros FC, Juan L, et al. Global epidemiology of use of and disparities in caesarean sections. The Lancet. 2018 2018/10/13/;392(10155):1341-8.

9. Cho CE, Norman M. Cesarean section and development of the immune system in the offspring. American Journal of Obstetrics and Gynecology. 2013 2013/04/01/;208(4):249-54.

10. Hansen S, Halldorsson TI, Olsen SF, Rytter D, Bech BH, Granstrom C, et al. Birth by cesarean section in relation to adult offspring overweight and biomarkers of cardiometabolic risk. International Journal of Obesity. 2018 2018/01/01;42(1):15-9.

11. Jakobsson HE, Abrahamsson TR, Jenmalm MC, Harris K, Quince C, Jernberg C, et al. Decreased gut microbiota diversity, delayed Bacteroidetes colonisation and reduced Th1 responses in infants delivered by Caesarean section. Gut. 2014;63(4):559.

12. Sandall J, Tribe RM, Avery L, Mola G, Visser GHA, Homer CSE, et al. Short-term and long-term effects of caesarean section on the health of women and children. The Lancet. 2018 2018/10/13/;392(10155):134957.

13. Nava-Gonzalez EJ, Gallegos-Cabriales EC, Leal-Berumen I, Bastarrachea RA. Mini-Review: The Contribution of Intermediate Phenotypes to GxE Effects on Disorders of Body Composition in the New OMICS Era. Int J Environ Res Public Health. 2017;14(9):1079.

14. Arnau-Soler A, Macdonald-Dunlop E, Adams MJ, Clarke T-K, MacIntyre DJ, Milburn K, et al. Genomewide by environment interaction studies of depressive symptoms and psychosocial stress in UK Biobank and Generation Scotland. Transl Psychiatry. 2019;9(1):14-.

15. Sudlow C, Gallacher J, Allen N, Beral V, Burton P, Danesh J, et al. UK biobank: an open access resource for identifying the causes of a wide range of complex diseases of middle and old age. PLoS Med. 2015;12(3):e1001779-e.

16. Hamilton GF, McDonald C, Chenier TC. Measurement of Grip Strength: Validity and Reliability of the Sphygmomanometer and Jamar Grip Dynamometer. Journal of Orthopaedic \& Sports Physical Therapy. 1992 1992/11/01;16(5):215-9.

17. UK Biobank: Grip-strength measurement using ACE. [cited; Available from:http://biobank.ndph.ox.ac.uk/showcase/refer.cgi?id=100232

18. Bycroft C, Freeman C, Petkova D, Band G, Elliott LT, Sharp K, et al. The UK Biobank resource with deep phenotyping and genomic data. Nature. 2018;562(7726):203-9.

19. Manichaikul A, Mychaleckyj JC, Rich SS, Daly K, Sale M, Chen W-M. Robust relationship inference in genome-wide association studies. Bioinformatics. 2010;26(22):2867-73.

20. Chang CC, Chow CC, Tellier LC, Vattikuti S, Purcell SM, Lee JJ. Second-generation PLINK: rising to the challenge of larger and richer datasets. Gigascience. 2015;4:7-.

21. Kraft P, Yen YC, Stram DO, Morrison J, Gauderman WJ. Exploiting Gene-Environment Interaction to Detect Genetic Associations. Human Heredity. 2007;63(2):111-9.

22. Watanabe K, Taskesen E, van Bochoven A, Posthuma D. Functional mapping and annotation of genetic associations with FUMA. Nat Commun. 2017;8(1):1826-. 
23. Liberzon A, Subramanian A, Pinchback R, Thorvaldsdottir H, Tamayo P, Mesirov JP. Molecular signatures database (MSigDB) 3.0. Bioinformatics. 2011;27(12):1739-40.

24. Bokulich NA, Chung J, Battaglia T, Henderson N, Jay M, Li H, et al. Antibiotics, birth mode, and diet shape microbiome maturation during early life. Sci Transl Med. 2016;8(343):343ra82-ra82.

25. Hyde MJ, Mostyn A, Modi N, Kemp PR. The health implications of birth by Caesarean section. Biological Reviews. 2012 2012/02/01;87(1):229-43.

26. Sharples AP, Polydorou I, Hughes DC, Owens DJ, Hughes TM, Stewart CE. Skeletal muscle cells possess a 'memory' of acute early life TNF- $\alpha$ exposure: role of epigenetic adaptation. Biogerontology. 2016 2016/06/01;17(3):603-17.

27. Nay K, Jollet M, Goustard B, Baati N, Vernus B, Pontones M, et al. Gut bacteria are critical for optimal muscle functiona potential link with glucose homeostasis. AJP Endocrinology and Metabolism. 2019 05/01;317.

28. Sire R, Rizzatti G, Ingravalle F, Pizzoferrato M, Petito V, Lopetuso L, et al. Skeletal muscle-gut axis: emerging mechanisms of sarcopenia for intestinal and extra intestinal diseases. Minerva gastroenterologica e dietologica. 2018 07/18;64.

29. Lahiri S, Kim H, Garcia-Perez I, Reza MM, Martin KA, Kundu P, et al. The gut microbiota influences skeletal muscle mass and function in mice. Sci Transl Med. 2019;11(502):eaan5662.

30. Korpela K, Salonen A, Vepsäläinen O, Suomalainen M, Kolmeder C, Varjosalo M, et al. Probiotic supplementation restores normal microbiota composition and function in antibiotic-treated and in caesareanborn infants. Microbiome. 2018;6(1):182-.

31. Grosicki GJ, Fielding RA, Lustgarten MS. Gut Microbiota Contribute to Age-Related Changes in Skeletal Muscle Size, Composition, and Function: Biological Basis for a Gut-Muscle Axis. Calcif Tissue Int. 2018;102(4):433-42.

32. Wampach L, Heintz-Buschart A, Fritz JV, Ramiro-Garcia J, Habier J, Herold M, et al. Birth mode is associated with earliest strain-conferred gut microbiome functions and immunostimulatory potential. Nat Commun. 2018;9(1):5091-.

33. Fulde M, Sommer F, Chassaing B, van Vorst K, Dupont A, Hensel M, et al. Neonatal selection by Toll-like receptor 5 influences long-term gut microbiota composition. Nature. 2018 2018/08/01;560(7719):489-93.

34. Delaney K, Kasprzycka P, Ciemerych MA, Zimowska M. The role of TGF- $\beta 1$ during skeletal muscle regeneration. Cell Biology International. 2017 2017/07/01;41(7):706-15.

35. Myers KA, Nasioulas S, Boys A, McMahon JM, Slater H, Lockhart P, et al. ADGRV1 is implicated in myoclonic epilepsy. Epilepsia. 2018 2018/02/01;59(2):381-8.

36. Urano T, Shiraki M, Yagi H, Ito M, Sasaki N, Sato M, et al. GPR98/Gpr98 Gene Is Involved in the Regulation of Human and Mouse Bone Mineral Density. The Journal of Clinical Endocrinology \& Metabolism. 2012;97(4):E565-E74.

37. Jules J, Ashley JW, Feng X. Selective targeting of RANK signaling pathways as new therapeutic strategies for osteoporosis. Expert Opin Ther Targets. 2010;14(9):923-34.

38. Langen RCJ, Schols AMWJ, Kelders MCJM, Wouters EFM, Janssen-Heininger YMW. Inflammatory cytokines inhibit myogenic differentiation through activation of nuclear factor- $x$ B. The FASEB Journal. 2001 2001/05/01;15(7):1169-80.

39. Bonnet N, Bourgoin L, Biver E, Douni E, Ferrari S. RANKL inhibition improves muscle strength and insulin sensitivity and restores bone mass. J Clin Invest. 2019;129(8):3214-23. 
40. Laurent MR, Dubois V, Claessens F, Verschueren SMP, Vanderschueren D, Gielen E, et al. Musclebone interactions: From experimental models to the clinic? A critical update. Molecular and Cellular Endocrinology. 2016 2016/09/05/;432:14-36.

41. McQueen C, Hughes GL, Pownall ME. Skeletal muscle differentiation drives a dramatic downregulation of RNA polymerase III activity and differential expression of Polr3g isoforms. Developmental Biology. 2019 2019/10/01/;454(1):74-84.

42. Dominguez R. Actin filament nucleation and elongation factors-structure-function relationships. Crit Rev Biochem Mol Biol. 2009 Nov-Dec;44(6):351-66.

43. Mitra P, Thanabalu T. Myogenic differentiation depends on the interplay of Grb2 and N-WASP. Biochimica et Biophysica Acta (BBA) - Molecular Cell Research. 2017 2017/03/01/;1864(3):487-97.

44. Cai G-Q, Chou C-F, Hu M, Zheng A, Reichardt LF, Guan J-L, et al. Neuronal Wiskott-Aldrich syndrome protein (N-WASP) is critical for formation of $\alpha$-smooth muscle actin filaments during myofibroblast differentiation. Am J Physiol Lung Cell Mol Physiol. 2012;303(8):L692-L702.

45. Cirak S, Foley AR, Herrmann R, Willer T, Yau S, Stevens E, et al. ISPD gene mutations are a common cause of congenital and limb-girdle muscular dystrophies. Brain. 2013;136(Pt 1):269-81.

46. Riemersma M, Froese DS, van Tol W, Engelke Udo F, Kopec J, van Scherpenzeel M, et al. Human ISPD Is a Cytidyltransferase Required for Dystroglycan O-Mannosylation. Chemistry \& Biology. 2015 2015/12/17/;22(12):1643-52.

47. Strecker P, Ludewig S, Rust M, Mundinger TA, Görlich A, Krächan EG, et al. FE65 and FE65L1 share common synaptic functions and genetically interact with the APP family in neuromuscular junction formation. Sci Rep. 2016;6:25652-.

48. Suh J, Moncaster JA, Wang L, Hafeez I, Herz J, Tanzi RE, et al. FE65 and FE65L1 amyloid precursor protein-binding protein compound null mice display adult-onset cataract and muscle weakness. FASEB J. 2015;29(6):2628-39.

49. Soerensen M, Li W, Debrabant B, Nygaard M, Mengel-From J, Frost M, et al. Epigenome-wide exploratory study of monozygotic twins suggests differentially methylated regions to associate with hand grip strength. Biogerontology. 2019;20(5):627-47.

50. Pandey R, Bakay M, Hain HS, Strenkowski B, Elsaqa BZB, Roizen JD, et al. CLEC16A regulates splenocyte and NK cell function in part through MEK signaling. PLoS One. 2018;13(9):e0203952-e.

51. Tam RCY, Li MWM, Gao YP, Pang YT, Yan S, Ge W, et al. Human CLEC16A regulates autophagy through modulating mTOR activity. Experimental Cell Research. 2017 2017/03/15/;352(2):304-12.

52. Cadwell K. Crosstalk between autophagy and inflammatory signalling pathways: balancing defence and homeostasis. Nature Reviews Immunology. 2016 2016/11/01;16(11):661-75.

53. Ilha J, do Espírito-Santo CC, de Freitas GR. mTOR Signaling Pathway and Protein Synthesis: From Training to Aging and Muscle Autophagy. In: Xiao J, editor. Muscle Atrophy. Singapore: Springer Singapore; 2018. p. 139-51.

54. Schiaffino S, Dyar KA, Ciciliot S, Blaauw B, Sandri M. Mechanisms regulating skeletal muscle growth and atrophy. The FEBS Journal. 2013 2013/09/01;280(17):4294-314.

55. Federico P, Perez MA. Distinct Corticocortical Contributions to Human Precision and Power Grip. Cereb Cortex. 2017;27(11):5070-82.

56. Kara M, Özçakar L, Kaymak B, Ata A, Frontera W. A "Neuromuscular Look" to sarcopenia: Is it a movement disorder? Journal of rehabilitation medicine. 2020 04/07;52.

Table1. Characteristics of the study population 


\begin{tabular}{lllll}
\hline Variables & ALL & FEMALE & MALE & P value \\
\hline HGS (left) N=160,635 & & & & \\
SEX, male & $66941,41.67 \%$ & & & \\
AGE, years & $55.84 \pm 7.71$ & $55.44 \pm 7.63$ & $56.40 \pm 7.78$ & $<0.001$ \\
BMI, kg/m & $26.73 \pm 4.55$ & $26.34 \pm 4.88$ & $27.27 \pm 3.97$ & $<0.001$ \\
HGS(left), kg & $29.88 \pm 10.99$ & $23.10 \pm 6.38$ & $39.37 \pm 8.85$ & $<0.001$ \\
C-section & $4371,2.72 \%$ & $2432,2.60 \%$ & $1939,2.90 \%$ & $<0.001$ \\
HGS (right) N=160,651 & HGS (right) $\mathbf{N = 1 6 0 , 6 5 1}$ & & & \\
SEX, male & $66939,41.67 \%$ & & & \\
AGE, years & $55.84 \pm 7.71$ & $55.44 \pm 7.63$ & $56.40 \pm 7.78$ & $<0.001$ \\
BMI, kg/m & $26.73 \pm 4.55$ & $26.34 \pm 4.89$ & $27.27 \pm 3.97$ & $<0.001$ \\
HGS(right), kg & $32.11 \pm 10.99$ & $25.37 \pm 6.43$ & $41.55 \pm 8.89$ & $<0.001$ \\
C-section & $4371,2.72 \%$ & $2432,2.60 \%$ & $1939,2.90 \%$ & $<0.001$ \\
\hline
\end{tabular}

\begin{tabular}{|c|c|c|c|}
\hline & Regression model of HGS (left) & Regression model of HGS (left) & Regression model of $\mathrm{HG}$ \\
\hline & $\beta$ & SE & T value \\
\hline C-section & -0.7700 & 0.1107 & -7.03 \\
\hline AGE, years & -0.2655 & 0.0024 & -112.63 \\
\hline SEX, male & 16.5271 & 0.0367 & 449.90 \\
\hline $\mathrm{BMI}, \mathrm{kg} / \mathrm{m}^{2}$ & 0.0197 & 0.0040 & 4.95 \\
\hline Adjusted $\mathbf{R}^{2}$ & 0.5699 & 0.5699 & 0.5699 \\
\hline
\end{tabular}

Table2. Regression analyses of HGS and C-section

Table3. Gene set enrichment analyses

\begin{tabular}{llll}
\hline Category & GeneSet & P value & Adjusted P value \\
\hline GO_bp & GO_CENTRAL_NERVOUS_SYSTEM_DEVELOPMENT & $2.69 \times 10^{-7}$ & $1.98 \times 10^{-3}$ \\
GO_bp & GO_HEAD_DEVELOPMENT & $1.38 \times 10^{-6}$ & $5.09 \times 10^{-3}$ \\
GO_bp & GO_CIRCULATORY_SYSTEM_DEVELOPMENT & $1.01 \times 10^{-5}$ & $2.48 \times 10^{-2}$ \\
GO_bp & GO_REGULATION_OF_INTRACELLULAR_SIGNAL_TRANSDUCTION & $1.45 \times 10^{-5}$ & $2.66 \times 10^{-2}$ \\
GO_cc & GO_CELL_JUNCTION & $4.36 \times 10^{-5}$ & $1.63 \times 10^{-2}$ \\
GO_cc & GO_SYNAPSE & $5.45 \times 10^{-5}$ & $1.63 \times 10^{-2}$ \\
GO_cc & GO_NEURON_PART & $5.59 \times 10^{-5}$ & $1.63 \times 10^{-2}$ \\
GO_cc & GO_CELL_LEADING_EDGE & $6.53 \times 10^{-5}$ & $1.63 \times 10^{-2}$ \\
GO_cc & GO_NEURON_PROJECTION & $1.87 \times 10^{-4}$ & $3.67 \times 10^{-2}$ \\
GO_cc & GO_PLASMA_MEMBRANE_REGION & $2.20 \times 10^{-4}$ & $3.67 \times 10^{-2}$ \\
GO_mf & GO_ENZYME_ACTIVATOR_ACTIVITY & $1.61 \times 10^{-5}$ & $2.65 \times 10^{-2}$ \\
GO_mf & GO_GTPASE_REGULATOR_ACTIVITY & $5.08 \times 10^{-5}$ & $4.18 \times 10^{-2}$ \\
\hline
\end{tabular}

Figure legend

Figure1. Genome-wide of environment interaction study of HGS

a), b) are the Manhattan plots of left HGS and right HGS, respectively. The blue solid line indicates the $\mathrm{P}$ value threshold for genome-wide significant $\left(P<5 \times 10^{-8}\right)$ while the red dotted line indicates the $\mathrm{P}$ value threshold for suggestive significance $\left(P<1 \times 10^{-5}\right)$. c) Gene-set enrichment analysis of HGS.

\section{Supplementary materials}


Supplementary Table S1. Identified SNPs interaction with C-section effecting on left HGS $\left(P<1.0 \times 10^{-5}\right)$ Supplementary Table S2. Supplement Table 2. Identified SNPs interaction with C-section effecting on right HGS $\left(P<1.0 \times 10^{-5}\right)$

Supplementary Table S3. Overlapped SNPs interaction with C-section $(\mathrm{P}<1.0 \times 10-5)$

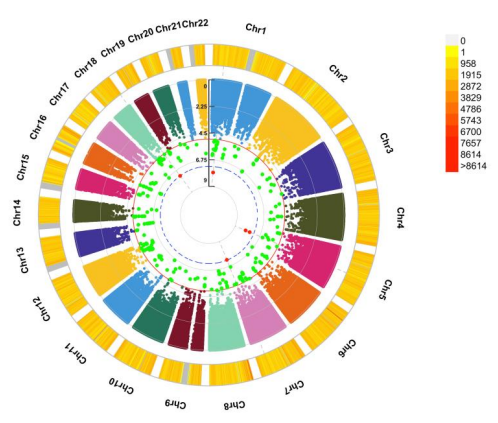

a)

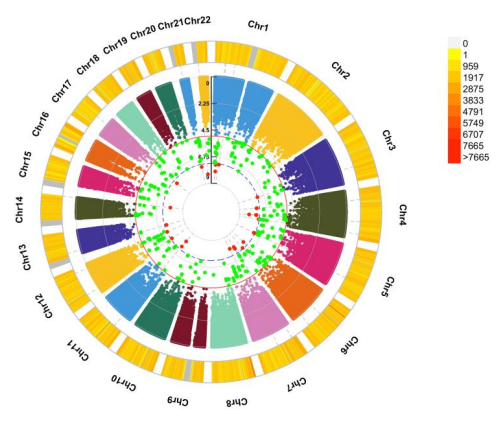

b)

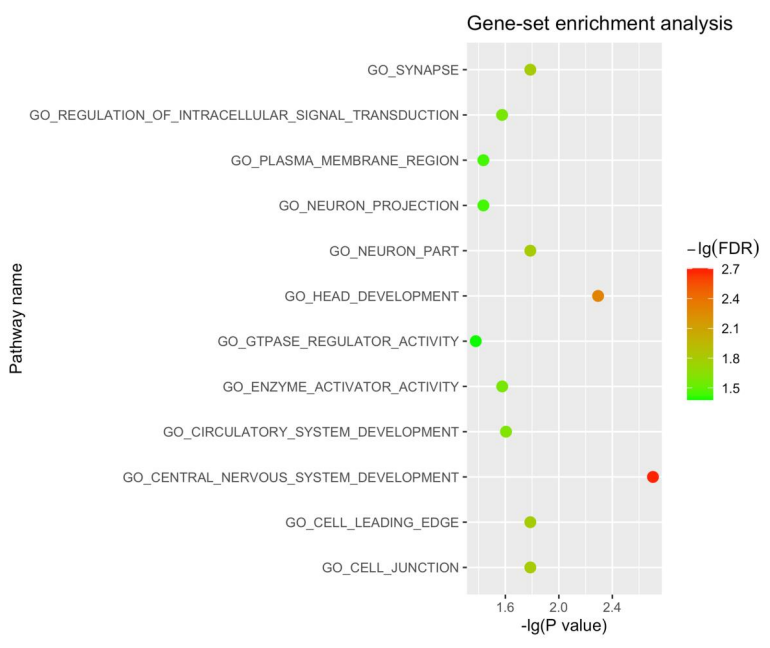

c) 